\title{
Testing the performance of a prototype lateral flow device using bronchoalveolar lavage fluid for the diagnosis of invasive pulmonary aspergillosis in high-risk patients
}

\author{
Caroline G. Castillo $^{1}$ | Carol A. Kauffman ${ }^{1,2}$ | Jingyi Zhai ${ }^{3}$ | Hui Jiang ${ }^{3}$ | \\ Stephanie M. Agozino ${ }^{4}$ | Marisa H. Miceli ${ }^{1}$ ii
}

${ }^{1}$ Division of Infectious Diseases, Department of Internal Medicine, University of Michigan Health System, Ann Arbor, MI, USA

${ }^{2}$ Veterans Affairs Ann Arbor Healthcare System, University of Michigan, Ann Arbor, MI, USA

${ }^{3}$ Department of Biostatistics, University of Michigan, Ann Arbor, MI, USA

${ }^{4}$ Clinical Microbiology Laboratory, University of Michigan Health System, Ann Arbor, MI, USA

\section{Correspondence}

Marisa H. Miceli, Division of Infectious Diseases, University of Michigan Medical School, Ann Arbor, MI, USA.

Email: mmiceli@umich.edu

\begin{abstract}
Summary
The diagnosis of invasive pulmonary aspergillosis (IPA) increasingly relies on nonculture-based biomarkers in bronchoalveolar lavage (BAL) fluid. The Aspergillus lateral flow device (LFD) is a rapid immunoassay that uses a novel Aspergillus monoclonal antibody to gain specificity. The objective of the study is to compare specificity and sensitivity of the prototype LFD and the galactomannan (GM) enzyme immunoassay in BAL fluid in high-risk patients. A total of 114 BAL samples from 106 patients at high risk for IPA were studied: 8 patients had proven/probable IPA, 16 had possible IPA and 82 did not have IPA. In patients with proven/probable IPA, specificity of LFD was $94 \%$ and GM was $89 \%$; sensitivity of LFD was $38 \%$ and GM was $75 \%$. Negative predictive value (NPV) for LFD was $94 \%$ and for GM was $98 \%$; positive predictive value (PPV) was $38 \%$ for both tests. The use of anti-mould prophylaxis did not affect specificity but resulted in decreased NPV of both LFD and GM. Union and intersection analysis showed no improvement in the performance by using both tests. Among patients at risk for IPA, the diagnostic performance of LFD and GM in BAL fluid appears comparable; specificity is high, but sensitivity of both LFD and GM is poor.
\end{abstract}

\section{KEYWORDS}

bronchoalveolar lavage, diagnostic test, invasive aspergillosis, lateral flow device, pulmonary aspergillosis

\section{1 | INTRODUCTION}

Invasive pulmonary aspergillosis (IPA) remains a deadly disease, with mortality rates as high as 35\%-57\% reported among haematopoietic cell transplant $(\mathrm{HCT})$ recipients, patients with acute leukaemia and liver transplant recipients. ${ }^{1-3}$ Prompt initiation of antifungal therapy improves survival, but early diagnosis remains challenging. ${ }^{4,5}$ To establish a diagnosis of proven IPA, clinicians are tasked with obtaining a sterile specimen or tissue for histopathology from which Aspergillus must be isolated or seen invading tissue. ${ }^{6}$ However, invasive procedures are often contraindicated in patients at greatest risk for this infection, and clinicians are often faced with the dilemma of starting empirical antifungal therapy.
Non-invasive diagnostic biomarkers, such as Aspergillus galactomannan (GM) and 1,3 beta-D-glucan (BDG), are included in the EORTC/MSG criteria for the diagnosis of probable IPA. The role of serum and bronchoalveolar lavage (BAL) GM in diagnosis has been best established for patients who have haematological malignancies. ${ }^{7}$ However, the use of serum and BAL GM in other populations, such as solid organ transplant recipients, remains undefined, ${ }^{8,9}$ and the serum BDG assay is not specific for Aspergillus.

The Aspergillus immunochromatographic assay (Isca Diagnostics, Truro, Cornwall, UK) is a prototype lateral flow device (LFD) that uses an Aspergillus-specific monoclonal antibody (mAb JF5) for the diagnosis of IPA. ${ }^{10}$ This assay enables point-of-care testing for the rapid diagnosis of IPA using technology similar to that used to diagnose 
pregnancy. ${ }^{11}$ Initial studies on the performance of LFD in BAL fluid demonstrated sensitivity and specificity as high as $100 \%$ and $95 \%$, respectively, ${ }^{12-14}$ but further clinical experience is needed to define the role of this assay for the diagnosis of IPA.

We sought to determine the utility of the Aspergillus LFD for the diagnosis of IPA in BAL samples from at-risk patients. We compared the performance characteristics of the Aspergillus LFD to that of GM in BAL fluid in these patients.

\section{2 | METHODS}

\section{1 | Patients}

This study was performed at the University of Michigan Health System, a 1000-bed tertiary care medical centre in southeastern Michigan. Approval was obtained from the University of Michigan Medical Center Institutional Review Board.

From September 1, 2014 through August 30, 2015, patients who were identified as being at risk for IPA and who had undergone bronchoscopy with BAL were included in the study. Patients considered at risk were those who had classic risk factors as defined by EORTC/MSG guidelines: neutropenia ( $\leq 500$ neutrophils/ $\mu \mathrm{L}$ ) for more than 10 days, allogeneic HCT, solid organ transplant, inherited severe immunodeficiency, prolonged use of corticosteroids ( $\geq 0.3 \mathrm{mg} / \mathrm{kg} /$ day prednisone equivalent $\geq 3$ weeks) or treatment with T-cell immunosuppressive agents, such as cyclosporine, tumour necrosis factor antagonists, specific monoclonal antibodies or nucleoside analogues, during the previous 3 months. ${ }^{6}$ Other atrisk patients included in the study were those who had a prolonged intensive care unit stay (>21 days), severe burns or near-drowning, autoimmune disease treated with immunosuppressive agents, HIV with CD4 count $<200 / \mu \mathrm{L}$, chronic pulmonary disease or solid tumour treated with chemotherapy in the preceding 30 days.

Exclusion criteria included age $<18$ years, inadequate BAL specimen (either due to quantity or quality of specimen) to perform GM and LFD testing and patients who had a BAL performed for a purpose other than evaluation for possible IPA. If patients had several BALs within a 14-day period, only the first specimen was included in this study. BAL samples from the same patient that were collected $>2$ weeks from the first BAL sample were tested and recorded as a separate episode.

Proven, probable and possible IPA were defined according to EORTC/MSG criteria. ${ }^{6}$ Additionally, the algorithm proposed by Blot et $\mathrm{al}^{15}$ was used to define IPA in non-neutropenic patients. For this study, biomarker detection in serum or BAL samples was not used as part of the diagnostic criteria for probable IPA. Information regarding host risk factors and diagnostic criteria for IPA was abstracted by chart review.

\subsection{Specimens and assays}

BAL samples were prospectively collected using normal saline from consecutive bronchoscopies performed during the study period. BAL samples were stored at $4^{\circ} \mathrm{C}$ for a maximum of $24 \mathrm{~h}$, and aliquots were prepared and frozen at $-20^{\circ} \mathrm{C}$. BAL samples from patients determined to be at risk for IPA, as defined above, were stored for LFD testing and GM testing, if not previously performed. BAL samples were not pretreated before freezing. Specimens remained frozen for a maximum of 7 months before testing.

Results of serum and BAL Aspergillus GM assays (Platelia ${ }^{\mathrm{TM}}$ Aspergillus EIA, Viracor-IBT Laboratories, Lee's Summit, MO, USA) obtained for clinical care were recorded. For patients who did not have GM testing performed previously on BAL fluid, frozen aliquots were sent for testing (Platelia ${ }^{\mathrm{TM}}$ Aspergillus EIA, Mira Vista Diagnostics, Indianapolis, IN, USA). Results with optical density index (ODI) values of $>0.5$ and $\geq 1.0$ were analysed separately.

The Aspergillus LFD was performed on stored BAL fluid following methods described previously. ${ }^{16}$ Prototype LFD devices were purchased from OLM Diagnostics, Newcastle upon Tyne, UK in April 2015. Testing was performed in two batches: the first in May 2015 and the second in September 2015. Results were interpreted independently by two investigators, both of whom were blinded to the source of the BAL. A positive result was indicated by the appearance of both a test band and a control band, and a negative result showed only the single control band. A positive test was reported as + (weak positive), ++ (positive) and +++ (strongly positive) as recommended by the manufacturer. If no control band was observed, the test results were considered invalid, and the test was repeated if ample specimen was available. If inadequate BAL fluid was available for retesting, the BAL sample was excluded from analysis.

\section{3 | Statistical methods}

Sensitivity, specificity, positive predictive value (PPV) and negative predictive value (NPV) for GM and LFD were calculated using $R$ package CARET. The differences in sensitivity and specificity between GM and LFD in the same patient group were tested using McNemar's test with function sesp.mcnemar in R package DTCOMPAIR. The differences in NPV and PPV between GM and LFD in the same patient group were tested using a weighted generalised score test with function pv.wgs in R package DTCOMPAIR. ${ }^{17}$ The differences in sensitivity, specificity, PPV and NPV for GM and LFD among different patient groups were tested using Fisher's exact test. In addition, we performed union and intersection analysis to determine whether the combination of both tests (LFD and GM) would impact performance in the diagnosis of IPA. For the union of two tests, the test result is negative if and only if both tests give negative results. For the intersection of two tests, the result is positive if and only if both tests give positive results.

\section{3 | RESULTS}

\section{1 | Patients and diagnoses}

We prospectively collected a total of $1211 \mathrm{BAL}$ samples. Of these, 1056 BAL samples from patients with low risk for IPA or age <18 
were excluded from the study. Forty-one additional specimens were excluded because of inadequate volume or missing clinical data.

A total of 114 BAL samples from 106 unique patients at risk for IPA were studied. Ninety-nine patients had one BAL sample, six patients had two BAL samples and one patient had three BAL samples included in the study. The mean age of the 106 patients was $55.3 \pm 16.8$ years (range 18-86 years), and 65\% were men. Clinical and demographic features of 106 patients included in the study are presented in Table 1.

For the $114 \mathrm{BAL}$ samples, the final diagnoses were proven IPA in 1, probable IPA in 7 samples from 7 patients and possible IPA in 18 samples from 16 patients by EORTC/MSG criteria. All seven cases of probable IPA met criteria for putative IPA according to the criteria proposed by Blot et al. None of 16 patients with possible IPA by the EORTC/MSG definition met diagnostic criteria for putative IPA by the Blot criteria.

An alternative diagnosis was established for the remaining 88 samples from 82 patients, including 4 for whom other invasive fungal infections (fusariosis in 2, mucormycosis in 1 and blastomycosis in 1) were present.

TAB LE 1 Clinical and demographic features of 106 patients included in the study

\begin{tabular}{|l|l|}
\hline Feature & $\begin{array}{c}\text { Number of } \\
\text { patients (\%) }\end{array}$ \\
\hline Age, mean \pm SD (years) & $55.3 \pm 16.8$ \\
\hline Men & $69(65.1)$ \\
\hline Classic risk factors ${ }^{\text {a }}$ & $71(67)$ \\
\hline Neutropenia & 10 \\
\hline Allogeneic haematopoietic cell transplant (HCT) & 15 \\
\hline Corticosteroids & 25 \\
\hline T-cell immunosuppression & 42 \\
\hline Chronic granulomatous disease & 1 \\
\hline Populations at risk based on underlying condition & \\
\hline Haematological cancer/transplant & $30(29)$ \\
\hline Non-HCT & 12 \\
\hline HCT/cord transplant & 18 \\
\hline Solid organ transplant & $24(22)$ \\
\hline Lung transplant & 15 \\
\hline Non-lung transplant & 9 \\
\hline Non-haematological/non-solid organ transplant & $52(49)$ \\
\hline Solid tumour on active chemotherapy & 4 \\
\hline Chronic pulmonary disease ${ }^{b}$ & 18 \\
\hline Critically ill (burn, trauma, ICU) & 9 \\
\hline Autoimmune on immunosuppressive drugs & 16 \\
\hline Other & 5 \\
\hline & \\
\hline & \\
\hline
\end{tabular}

${ }^{\text {aS }}$ everal patients had more than one classic risk factor.

${ }^{b}$ Eight patients were on high-dose corticosteroids and 10 were on lowdose corticosteroids with or without inhaled corticosteroids.

'Other conditions included diabetes mellitus (1), chronic heart disease secondary to congenital heart disease (1), HIV infection with CD4 <200/ $\mu \mathrm{L}$ (1), asthma and relapsed solid organ tumour (not on active chemotherapy) (1) and sickle cell anaemia (1).

\subsection{Performance of LFD and GM in BAL fluid}

Among the eight BAL samples from patients with proven/probable IPA, the LFD assay was positive in only three. A positive GM assay with an ODI cut-off $\geq 0.5$ (GM0.5) was found in six BAL samples. Only three out of eight BAL samples tested positive by both LFD and GM0.5 assays (Table 2). Eighteen BAL samples were from patients with possible IPA. The LFD assay was positive in two of these samples, and the GM0.5 was positive in a third sample.

Among the 88 BAL samples from patients who did not have IPA, there were 83 negative and 5 positive LFD results. GM0.5 was negative in 78 and positive in 10 samples. There were more true negative test results with LFD when compared with GM0.5.

For proven/probable IPA, the specificity of LFD and GM0.5 was $94 \%$ and $89 \%$ respectively (Table 3 ). The sensitivity of GM0.5 was higher (75\%) than that of LFD (38\%). The NPV for LFD and GM0.5 tests was $94 \%$ and $98 \%$ respectively. The PPV was $38 \%$ for both LFD and GM0.5 (Table 3).

When cases of proven/probable and possible IPA were included, the specificity of LFD and GM0.5 was $94 \%$ and $89 \%$ respectively (Table 3). The sensitivity of GM0.5 was slightly higher (27\%) than that of LFD (19\%). The NPV for both LFD and GM0.5 tests was $80 \%$. The PPV was low for both LFD (50\%) and GM0.5 (41\%).

Analysis of the performance of GM0.5 and LFD in the different subgroups of patients at risk for IPA overall showed high specificity and low sensitivity in all groups (Table 4).

\subsection{Performance of GM using an ODI cut-off of $\geq 1.0$}

When the GM cut-off was increased to an ODI of $\geq 1.0$ (GM1.0), the number of positive tests was 13 , compared with 17 when the cutoff was $\geq 0.5$; there were 7 false positive tests with GM1.0 compared with 10 false positive tests with GM0.5. Using the GM1.0 cut-off, increased specificity only slightly from $89 \%$ to $92 \%$, and the sensitivity decreased from $75 \%$ to $62 \%$ when proven/probable cases were considered (Table 3). Increasing the GM cut-off point to 1.0 did not improve performance of the assay in specific patient groups (data not shown).

\section{4 | Combination LFD and GM testing}

We performed union and intersection analyses to determine whether the combination of LFD and GM0.5 would impact performance for the diagnosis of IPA (Table 3). Obtaining a positive result with either the GM0.5 or LFD assay (union analysis), retained the sensitivity of the individual test with the highest sensitivity, in this case GM0.5, and was more sensitive than using the LFD test alone. Union analysis resulted in a decrease in specificity (86\%) compared with the specificity of either test alone ( $94 \%$ and $89 \%$ for LFD and GM0.5 respectively). Intersection analysis in which both assays had to yield a positive result predictably increased the specificity but only slightly because the specificity of the individual assays was high to begin with. The sensitivity was only $38 \%$. Thus, little was gained by performing both assays. 
TABLE 2 LFD and GM results in patients with proven/probable invasive pulmonary aspergillosis

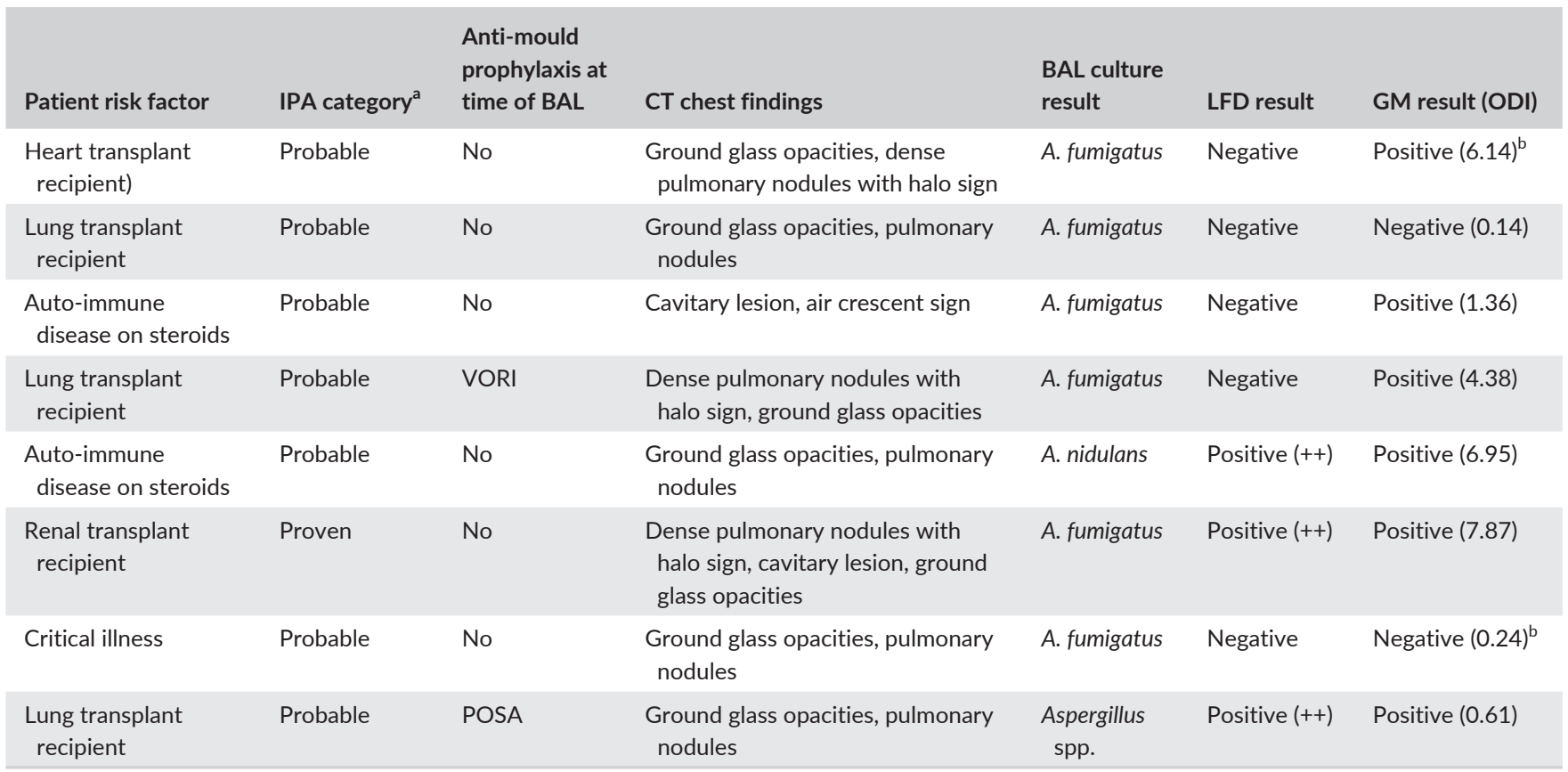

BAL, bronchoalveolar lavage; GM, galactomannan; IPA, invasive pulmonary aspergillosis; LFD, lateral flow device; ODI, optic density index; POSA, posaconazole; VORI, voriconazole.

${ }^{a}$ Patients met the definition of probable IPA by EORTC/MSG criteria ${ }^{6}$ and also met the definition of putative IPA by the criteria proposed by Blot et al ${ }^{15}$. ${ }^{\mathrm{b}} \mathrm{BAL}$ GM testing performed retrospectively.

\subsection{Effect of mould-active prophylaxis on test performance}

A total of 27 BAL samples were obtained from 24 patients who were receiving anti-Aspergillus prophylaxis with voriconazole, posaconazole, itraconazole, micafungin or aerosolised or intravenous amphotericin B. Two of eight samples were from patients who had proven/probable IPA and who were receiving prophylaxis. GM0.5 was positive in both samples, and LFD was positive in one. Ten samples from nine patients with possible IPA were obtained while they were receiving prophylaxis. The GM0.5 and LFD test were positive in only one patient each. Among 15 samples from 13 patients who did not have IPA and who were on prophylaxis, GM0.5 was positive in 2 and LFD was negative in all of them.

The specificity of LFD and GM0.5 was not influenced by antimould prophylaxis. The specificity of GM0.5 was $87 \%$ and $89 \%$ among patients with and without prophylaxis, respectively, and the specificity of LFD was $100 \%$ and $93 \%$ among patients with and without prophylaxis respectively. The NPV was decreased by the use of anti-mould prophylaxis from $86 \%$ to $60 \%$ with the LFD assay and from $87 \%$ to $59 \%$ using the GM0.5 assay.

\subsection{Cross-reactivity of LFD and GM assays}

Cultures of BAL samples yielded fungal organisms in 38 samples from patients who did not have IPA (Table S1). Cross-reaction with either the GM0.5 or the LFD assay was observed in 6 of the 38 samples.
There was a positive LFD test, but a negative GM0.5 test and a negative LFD test, but a positive GM0.5 test noted in two samples that yielded Penicillium spp. Two BAL samples that yielded colonising Aspergillus species, A. ochraceous and A. versicolor that were not associated with IPA, were both positive by LFD, but only one was positive by $\mathrm{GM} 0.5$.

A sample with Mucorales gave a positive GM0.5 test, but a negative LFD test. The BAL sample that yielded Blastomyces dermatiditis gave a negative LFD test, but a positive GM0.5 test. Both assays were negative in two samples that yielded Fusarium spp.

\section{4 | DISCUSSION}

We evaluated the diagnostic performance of the prototype Aspergillus LFD and GM assays on BAL samples from patients who had high pretest probability for IPA. We found that the performance of the prototype LFD assay for the diagnosis of IPA was comparable, but not superior, to that of the GM assay.

The prototype LFD assay has been studied in different populations at risk, including patients with haematological malignancies, solid organ transplant recipients, critically ill patients in the ICU and patients with chronic pulmonary disease. ${ }^{12-14,16,18-20}$ These studies varied with regard to the populations studied and the diagnostic criteria used to define IPA. Some included GM in BAL fluid as a diagnostic criterion, and others excluded patients who had possible IPA. The effect of anti-mould prophylaxis on test performance was generally not taken into account. The conclusion from these 
TAB LE 3 Performance of LFD and GM in BAL samples from patients at high risk of invasive pulmonary aspergillosis (IPA): single assay vs combination of assays (union and intersection analyses)

\begin{tabular}{|c|c|c|c|c|c|}
\hline Analysis & Test & Sensitivity \% (95\% Cl) & Specificity \% (95\% Cl) & PPV \% (95\% Cl) & NPV \% $(95 \% \mathrm{CI})$ \\
\hline \multicolumn{6}{|c|}{ Proven/probable vs non-IPA } \\
\hline \multirow[t]{2}{*}{ Individual assay } & LFD & $38(9,76)$ & $94(87,98)$ & $38(9,76)$ & $94(87,98)$ \\
\hline & GM1.0 & $62(24,91)$ & $92(84,97)$ & $42(15,72)$ & $96(90,99)$ \\
\hline Union & GM0.5 or LFD & $75(35,97)$ & $86(77,93)$ & $33(13,59)$ & $97(91,100)$ \\
\hline \multirow[t]{2}{*}{ Intersection } & GM0.5 and LFD & $38(9,76)$ & $97(90,99)$ & $50(12,88)$ & $94(88,98)$ \\
\hline & GM1.0 and LFD & $25(3,65)$ & $97(90,99)$ & $40(5,85)$ & $93(86,98)$ \\
\hline \multicolumn{6}{|c|}{ Proven/probable/possible vs non-IPA } \\
\hline \multirow[t]{2}{*}{ Individual assay } & LFD & $19(7,39)$ & $94(87,98)$ & $50(19,81)$ & $80(71,87)$ \\
\hline & GM0.5 & $27(12,48)$ & $89(80,94)$ & $41(18,67)$ & $80(71,88)$ \\
\hline Union & GM1.0 or LFD & $35(17,56)$ & $90(81,95)$ & $50(26,74)$ & $82(73,89)$ \\
\hline \multirow[t]{2}{*}{ Intersection } & GM0.5 and LFD & $12(2,30)$ & $97(90,99)$ & $50(12,88)$ & $79(70,86)$ \\
\hline & GM1.0 and LFD & $8(1,25)$ & $97(90,99)$ & $40(5,85)$ & $78(69,85)$ \\
\hline
\end{tabular}

BAL, bronchoalveolar lavage; GM0.5, galactomannan cut-off $\geq 0.5$; GM1.0, galactomannan cut-off $\geq 1.0$; LFD, lateral flow device; NPV, negative predictive value; PPV, positive predictive value. Union analysis implies that either GM or LFD should be positive for the diagnosis of invasive pulmonary aspergillosis. Intersection analysis implies that both GM and LFD should be positive for the diagnosis of invasive pulmonary aspergillosis.

\begin{tabular}{|c|c|c|c|c|c|c|c|}
\hline \multirow[b]{2}{*}{ Risk group } & \multirow{2}{*}{$\begin{array}{l}\text { Number of } \\
\text { BAL tested (\%) }\end{array}$} & \multicolumn{3}{|c|}{ Sensitivity } & \multicolumn{3}{|c|}{ Specificity } \\
\hline & & LFD & GM0.5 & $P$ value & LFD & GM0.5 & $P$ value \\
\hline $\begin{array}{l}\text { Classical risk factors } \\
\text { for IPA }\end{array}$ & 78 & 14 & 22 & .32 & 98 & 93 & .08 \\
\hline $\begin{array}{l}\text { Non-classical risk } \\
\text { factors for IPA }\end{array}$ & 36 & 50 & 50 & NA & 88 & 81 & .32 \\
\hline $\begin{array}{l}\text { Haematological } \\
\text { diseases }\end{array}$ & 33 & 7 & 7 & NA & 100 & 100 & NA \\
\hline $\begin{array}{l}\text { Solid organ } \\
\text { transplant }\end{array}$ & 27 & 33 & 67 & .32 & 95 & 90 & .32 \\
\hline $\begin{array}{l}\text { Non-haematological/ } \\
\text { non-transplant }\end{array}$ & 54 & 33 & 33 & NA & 92 & 83 & .16 \\
\hline
\end{tabular}

TABLE 4 Test performance in BAL for the diagnosis of proven/probable invasive pulmonary aspergillosis (IPA) in different subgroups of patients based on their risk

NA, not applicable.

studies was that prototype LFD had high specificity and NPV, but only modest sensitivity and PPV. However, a recent review noted sensitivity as high as $73 \%$ when results from the various studies were combined. ${ }^{21}$

Biomarkers for the diagnosis of IPA appeared to perform better in prior studies when used in patients who have haematological malignancies or have received a $\mathrm{HCT}^{7,8}$ However, the variability of these tests has been noted in other patient populations at risk for IPA. A recent meta-analysis found that the performance of GM in BAL fluid showed large variability in performance depending on the population studied and the cut-off value used. ${ }^{22}$ For this reason, we evaluated the performance of both tests in different population groups stratified according to their risk for IPA.
Specificity of the prototype LFD and GM0.5 assays was similar in the various subgroups that were studied, including solid organ transplant recipients. These findings differ from prior studies using BAL fluid that showed that the prototype LFD assay provided improved specificity when compared with the GM assay. ${ }^{14,16}$ This could be in part due to the large number of lung transplant recipients and the large number of false positive GM tests in BAL fluid reported in both of those prior studies.

Prior reports have shown that increasing the cut-off value for the GM assay to an ODI $\geq 1.0$ improved specificity and only slightly decreased sensitivity. ${ }^{13,22}$ We found little difference comparing the two cut-off points, and the higher cut-off had no impact on the performance of GM overall or in specific patient groups. 
We noted that the prototype LFD assay showed only a nonsignificant trend towards higher specificity when compared with that of GM0.5 and GM1.0. There are two potential explanations for this finding. First, most of the patients in the group with haematological malignancies or HCT had possible IPA and were receiving anti-mould prophylaxis at the time the BAL was performed; this likely led to negative fungal cultures and negative results with the GM and LFD assays. Second, most patients in our study with proven/probable IPA did not have a haematological malignancy or $\mathrm{HCT}$, and were from populations in which both GM and LFD assays have demonstrated variable performance. $^{8}$

Data on the effect of anti-mould drugs on the performance of biomarker testing for the diagnosis of IPA are conflicting. ${ }^{19,20,23-26}$ Eigl et $\mathrm{al}^{19}$ reported on the effect of prophylaxis among 60 patients with proven/probable IPA, noting a significant decrease in the sensitivity of prototype LFD and GM assays performed on BAL fluid of patients taking anti-mould prophylaxis. Similar findings have been noted on the performance of the GM assay on serum. ${ }^{25,26}$ In our study, the use of anti-mould agents at the time of bronchoscopy did not affect specificity of either test, but did, however, significantly decrease the NPV of both assays. This observation denotes an important limitation for both tests.

Molecular techniques, such as real-time PCR, have become increasingly useful in the diagnosis of IPA and are likely to be included as microbiological criteria in the upcoming revision of the EORTC/ MSG Guidelines. ${ }^{27,28}$ Several authors have reported on the use of a combination of biomarkers, including PCR, to enhance the diagnosis of IPA. ${ }^{13,20,29-31}$ Johnson et $\mathrm{al}^{20}$ found good agreement between the prototype LFD and PCR on BAL fluid from 32 patients, a third of whom had probable/proven IPA. Hoenigl et $\mathrm{al}^{13}$ using BAL specimens from high-risk patients showed that combining GM1.0 with prototype LFD or PCR resulted in an increase in sensitivity to $94 \%$ and $100 \%$ respectively. We found that using a combination of both prototype LFD and GM0.5 led to only a slight increase in sensitivity over the individual assays. The intersection analysis in which both assays had to yield a positive result predictably increased the specificity, but only slightly, and led to very low sensitivity.

False positive LFD results and cross-reactivity with other fungal antigens have been previously noted. Based on early in vitro studies, the mAb JF5 used in the prototype LFD could cross-react with antigens from Paecilomyces variotii and Penicillium spp. ${ }^{10}$ False positive LFD results have been reported previously with Penicillium spp. and occurred with one BAL sample in our study. ${ }^{12,14}$ Additionally, colonising non-pathogenic Aspergillus spp. not thought to be causing disease, give positive results with the LFD assay. ${ }^{12,14}$

A major limitation to our study is the small number of patients who had proven/probable IPA. Unfortunately, this limitation is commonly encountered in studies dealing with IPA and other invasive fungal infections. The incidence of proven/probable IPA in our cohort was similar to that previously reported in other multicentre studies. ${ }^{3,32}$ However, because we excluded biomarkers as microbiological criterion for the diagnosis of IPA, it is conceivable that some patients with probable IPA may have been classified as possible IPA. Another limitation is that we included a small number of patients for whom several samples, collected $>2$ weeks apart, were studied, perhaps weakening the analysis. Our study was conducted in a single centre, and thus, our findings and conclusions may not be applicable to other centres.

Although the incidence of IPA appears to be decreasing with the implementation of preventative measures, such as antifungal prophylaxis in high-risk patients, IPA remains a fatal disease if not promptly diagnosed and treated. For this reason, it is important to develop new tools for the diagnosis of IPA and to evaluate their application in the real world of clinical medicine. When the prototype LFD test was developed, it was perceived as a useful addition to the diagnostic armamentarium of IPA, and early studies were promising. It is inexpensive, simple to perform and results are available in minutes. These features made it a very attractive point of care test to help clinicians screen for IPA so that prompt intervention could ensue. However, our results confirm the findings of other studies, which indicated that the sensitivity of the LFD in BAL fluid may be lower than desired. With current advances in molecular technology, it is likely that a more sensitive test may be developed that utilises the highly specific Aspergillus JF5 monoclonal antibody that forms the basis for the current LFD assay.

\section{ACKNOWLEDGMENTS}

We thank Amy Drouillard and Deborah Soldner from the Microbiology Laboratory at the University of Michigan Health Center and Kathleen Symons from the Infectious Diseases Research Laboratory at the VA Ann Arbor Healthcare System for all their help and support in this project.

\section{CONFLICT OF INTEREST}

The authors have no conflicts of interest to declare.

\section{ORCID}

Marisa H. Miceli (iD http://orcid.org/0000-0002-3175-0512

\section{REFERENCES}

1. Lortholary O, Gangneux JP, Sitbon K, et al. Epidemiological trends in invasive aspergillosis in France: the SAIF networks (2005-2007). Clin Microbiol Infect. 2011;17:1882-1889.

2. Baddley JW, Andes DR, Marr KA, et al. Factors associated with mortality in transplant patients with invasive aspergillosis. Clin Infect Dis. 2010;50:1559-1567.

3. Neofytos D, Horn D, Anaissie E, et al. Epidemiology and outcome of invasive fungal infection in adult hematopoietic stem cell transplant recipients: analysis of multicenter prospective antifungal therapy (PATH) alliance registry. Clin Infect Dis. 2009;48:265-273.

4. Segal BH. Aspergillosis. N Engl J Med. 2009;360:1870-1884.

5. Gregg KS, Kauffman CA. Invasive aspergillosis: epidemiology, clinical aspects, and treatment. Semin Respir Crit Care Med. 2015;36:662-672.

6. De Pauw B, Walsh TJ, Donnelly JP, et al. Revised definitions of invasive fungal disease from the European Organization for Research and Treatment of Cancer/Invasive Fungal Infections Cooperative Group and the National Institute of Allergy and Infectious Diseases 
Mycoses Study Group (EORTC/MSG) Consensus Group. Clin Infect Dis. 2008;46:1813-1821.

7. Mennink-Kersten MA, Verweij PE. Non-culture based diagnostics for the opportunistic fungi. Infect Dis Clin North Am. 2006;20:711-727.

8. Pfeiffer C, Fine JP, Safdar M. Diagnosis of invasive aspergillosis using a galactomannan assay: a meta-analysis. Clin Infect Dis. 2006;42:1417-1427.

9. Miceli MH, Maertens J. Role of non-culture based tests, with an emphasis on galactomannan testing for the diagnosis of invasive aspergillosis. Semin Respir Crit Care Med. 2015;36:650-661.

10. Thornton CR. Development of an immunochromatographic lateralflow device for rapid serodiagnosis of invasive aspergillosis. Clin Vaccine Immunol. 2008;15:1095-1105.

11. Thornton CR. Detection of invasive aspergillosis. Adv Appl Microbiol. 2010;70:187-216.

12. Hoenigl M, Koidl C, Duettmann W, et al. Bronchoalveolar lavage lateral-flow device test for invasive pulmonary aspergillosis diagnosis in haematological malignancy and solid organ transplant patients. $J$ Infect. 2012;65:588-591.

13. Hoenigl M, Prattes J, Spiess B, et al. Performance of galactomannan, beta-d-glucan, Aspergillus lateral-flow device, conventional culture, and PCR tests with bronchoalveolar lavage fluid for diagnosis of invasive pulmonary aspergillosis. J Clin Microbiol. 2014;52:2039-2045.

14. Willinger B, Lackner M, Lass-Florl C, et al. Bronchoalveolar lavage lateral-flow device test for invasive pulmonary aspergillosis in solid organ transplant patients: a semiprospective multicenter study. Transplantation. 2014;98:898-902.

15. Blot SI, Taccone FS, Van den Abeele AM, et al. A clinical algorithm to diagnose invasive pulmonary aspergillosis in critically ill patients. Am J Respir Crit Care Med. 2012;186:56-64.

16. Miceli MH, Goggins MI, Chander P, et al. Performance of lateral flow device and galactomannan for the detection of Aspergillus species in bronchoalveolar fluid of patients at risk for invasive pulmonary aspergillosis. Mycoses. 2015;58:368-374.

17. Kosinski AS. A weighted generalized score statistic for comparison of predictive values of diagnostic tests. Stat Med. 2013;32:964-977.

18. Prattes J, Lackner M, Eigl S, et al. Diagnostic accuracy of the Aspergillus-specific bronchoalveolar lavage lateral-flow assay in haematological malignancy patients. Mycoses. 2015;58:461-469.

19. Eigl S, Prattes J, Reinwald M, et al. Influence of mould-active antifungal prophylaxis on the performance of the Aspergillus-specific bronchoalveolar lavage fluid lateral-flow device test. Int J Antimicrob Agents. 2015;46:401-405.

20. Johnson G, Sarker S-J, Nannini F, et al. Aspergillus-specific lateral-flow device and real-time PCR testing of bronchoalveolar lavage fluid: a combination biomarker approach for clinical diagnosis of invasive pulmonary aspergillosis. J Clin Microbiol. 2015;53:2103-2108.

21. Heldt $\mathrm{S}$, Hoenigl M. Lateral flow assays for the diagnosis of invasive aspergillosis: current status. Curr Fungal Infect Rep. 2017;11:45-51.

22. Zou M, Tang L, Zhao S, et al. Systematic review and meta-analysis of detecting galactomannan in bronchoalveolar lavage fluid for diagnosing invasive aspergillosis. PLoS ONE. 2012;7:e43347.

23. Reinwald $M$, Hummel $M$, Kovalevskaya $E$, et al. Therapy with antifungals decreases the diagnostic performance of PCR for diagnosing invasive aspergillosis in bronchoalveolar lavage samples of patients with haematological malignancies. J Antimicrob Chemother. 2012;67:2260-2267.

24. Musher B, Fredricks D, Leisenring W, Balajee SA, Smith C, Marr KA. Aspergillus galactomannan enzyme immunoassay and quantitative PCR for diagnosis of invasive aspergillosis with bronchoalveolar lavage fluid. J Clin Microbiol. 2004;42:5517-5522.

25. Marr KA, Laverdiere M, Gugel A, Leisenring W. Antifungal therapy decreases sensitivity of the Aspergillus galactomannan enzyme immunoassay. Clin Infect Dis. 2005;40:1762-1769.

26. Duarte RF, Sanchez-Ortega I, Cuesta I, et al. Serum galactomannanbased early detection of invasive aspergillosis in hematology patients receiving effective anti-mold prophylaxis. Clin Infect Dis. 2014;59:1696-1702.

27. White PL, Wingard JR, Bretagne S, et al. polymerase chain reaction: systematic review of evidence for clinical use in comparison with antigen testing. Clin Infect Dis. 2015;61:1293-1303.

28. Mengoli C, Cruciani M, Barnes RA, Loeffler J, Donnelly JP. Use of PCR for diagnosis of invasive aspergillosis: systematic review and metaanalysis. Lancet Infect Dis. 2009;9:89-96.

29. Avni T, Levy I, Sprecher H, Yahav D, Leibovici L, Paul M. Diagnostic accuracy of PCR alone compared to galactomannan in bronchoalveolar lavage fluid for diagnosis of invasive pulmonary aspergillosis: a systematic review. J Clin Microbiol. 2012;50:3652-3658.

30. Luong M-L, Clancy CJ, Vadnerkar A, et al. Comparison of an Aspergillus real-time polymerase chain reaction assay with galactomannan testing of bronchoalveolar lavage fluid for the diagnosis of invasive pulmonary aspergillosis in lung transplant recipients. Clin Infect Dis. 2011;52:1218-1226.

31. Arvanitis M, Anagnostou T, Mylonakis E. Galactomannan and polymerase chain reaction-based screening for invasive aspergillosis among high-risk hematology patients: a diagnostic meta-analysis. Clin Infect Dis. 2015;61:1263-1272.

32. Kontoyiannis DP, Marr KA, Park BJ, et al. Prospective surveillance for invasive fungal infections in hematopoietic stem cell transplant recipients, 2001-2006: overview of the TRANSNET database. Clin Infect Dis. 2010;50:1091-1100.

\section{SUPPORTING INFORMATION}

Additional Supporting Information may be found online in the supporting information tab for this article.

How to cite this article: Castillo CG, Kauffman CA, Zhai J, Jiang $\mathrm{H}$, Agozino SM, Miceli MH. Testing the performance of a prototype lateral flow device using bronchoalveolar lavage fluid for the diagnosis of invasive pulmonary aspergillosis in high-risk patients. Mycoses. 2018;61:4-10. https://doi.org/10.1111/ myc.12694 